\title{
CALIBRAÇÃO DO MODELO REGIONAL BRAMS PARA A PREVISÃO DE EVENTOS CLIMÁTICOS EXTREMOS
}

\author{
JULLIANA LARISE M. FREIRE, SAULO RIBEIRO DE FREITAS, \\ CAIO AUGUSTO DOS SANTOS COELHO
}

\author{
Instituto Nacional de Pesquisas Espaciais, Centro de Previsão de Tempo e Estudos Climáticos \\ (INPE/CPTEC), Cachoeira Paulista, SP, Brasil
}

julliana.freire@cptec.inpe.br, saulo.freitas@cptec.inpe.br, caio.coelho@cptec.inpe.br

Recebido Fevereiro de 2014 - Aceito Agosto de 2014

\begin{abstract}
RESUMO
As previsões climáticas sazonais têm contribuído efetivamente para o planejamento de atividades de diversos setores da sociedade, tais como, energia, agricultura e saúde. A necessidade de um maior detalhamento das previsões, através da diminuição da escala espacial da informação climática, tem concentrado nos últimos anos esforços científicos em várias regiões do globo. A aplicação da técnica de redução de escala através da modelagem dinâmica regional, conhecida como downscaling dinâmico, vem sendo utilizada com esse objetivo. Desta forma, o presente trabalho teve como objetivo calibrar e avaliar o modelo regional BRAMS, aninhado unidirecionalmente ao modelo global MCGA/CPTEC, em prever os eventos climáticos extremos de 1983 (El Niño) e 1989 (La Niña) sobre a América do Sul (AS), para o trimestre março-abril-maio (MAM). As previsões com o BRAMS foram produzidas na resolução espacial de $30 \mathrm{~km}$, tendo como condições de contorno a temperatura da superfície do mar persistida e a umidade do solo climatológica. Após o processo de calibração, o modelo BRAMS conseguiu capturar o padrão de precipitação associada aos eventos climáticos extremos, como a redução (aumento) da precipitação principalmente no leste da região Norte e Nordeste do Brasil e o aumento (redução) de precipitação na região Sul para o ano e 1983 (1989).
\end{abstract}

Palavras-chave: Downscaling; ENOS; previsão sazonal.

\begin{abstract}
CALIBRATION OF THE BRAMS REGIONAL MODEL FOR PREDICTING CLIMATE EXTREME EVENTS

The seasonal climate predictions have effectively contributed to the planning on various sectors of society, such as energy, agriculture and health activities. The need for more detailed forecasts by increasing spatial resolution climate information has concentrated research efforts recently in various regions of the globe. Downscaling technique by regional dynamic modeling, known as dynamic downscaling, has been used for this purpose. Thus, the present study aimed to evaluate and calibrate the regional BRAMS model, unidirectionally nested with AGCM/CPTEC global model, in predicting the extreme weather events of 1983 (El Niño) and 1989 (La Niña) over South America (SA) for March-April-May (MAM). The predictions were produced at spatial resolution of $30 \mathrm{~km}$, having the persisted sea surface temperature and climatological soil moisture as boundary conditions. After the calibration process, the BRAMS was able to capture the rainfall pattern associated with extreme weather events, such as the decrease (increase) in precipitation pattern mainly on the eastern of North region and Northeast Brazil, and the increase (decrease) of rainfall in the Southern region for the year 1983 (1989).
\end{abstract}

Keywords: Downscaling, ENSO, seasonal climate predictions 


\section{INTRODUÇÃO}

A previsão climática na escala de tempo sazonal (previsões para os próximos 1 a 6 meses) tem contribuído efetivamente para o planejamento de atividades de diversos setores da sociedade. As pesquisas da área de previsão climática vêm avançando para tentar atender as demandas da sociedade, que é afetada pela variabilidade climática em setores essenciais, tais como, energia, agricultura e saúde. A utilização eminente dos resultados das previsões climáticas nos setores citados tem exigido o desenvolvimento de técnicas ou métodos, que aprimorem o prognóstico de variáveis nas escalas temporal e espacial.

A habilidade em prever anomalias climáticas na escala de tempo sazonal tem sua origem nas condições da superfície da terra que apresentam variações lentas e podem influenciar o clima, tais como temperatura da superfície do mar (TSM), umidade do solo e cobertura de neve. Estas condições de contorno da superfície do planeta constituem a base para a previsão climática sazonal. Tais influências são pouco notadas em eventos meteorológicos diários, porém, se tornam perceptíveis em médias meteorológicas de longo prazo. As condições de superfície mais importantes que modulam a variabilidade do clima na escala de tempo sazonal são a TSM e a umidade do solo, sendo a primeira mais importante nas zonas tropicais. As previsões climáticas sazonais são mais bem sucedidas, apresentando maior destreza, nos trópicos devido a forte relação entre a TSM e as condições climáticas sazonais da região tropical (Goddard et al., 2001). A variação temporal da TSM pode ser representada em modelos computacionais da atmosfera e do oceano, modelos os quais formam a base dinâmica para a previsão climática sazonal.

A previsão do fenômeno acoplado oceano-atmosfera El Niño/Oscilação Sul (ENOS) é a mais bem sucedida em termos climáticos. A componente atmosférica desse fenômeno é representada pela Oscilação Sul, e pode ser quantificada pelo Índice de Oscilação Sul (IOS). Este índice representa a diferença de pressão ao nível do mar entre o Pacífico Central (Taiti) e o Pacífico Oeste (Darwin/Austrália). Valores negativos (positivos) elevados do IOS são indicadores da ocorrência de El Niño (La Niña).

El Niño e La Niña constituem a componente oceânica do fenômeno ENOS. As diferentes fases desses fenômenos climáticos de grande escala são caracterizadas por padrões anômalos de temperatura da superfície do mar no Oceano Pacífico, que ocorrem simultaneamente com anomalias de pressão em Taiti e Darwin. Esses fenômenos afetam a circulação atmosférica, resultando em anomalias pluviométricas em diversas regiões do globo terrestre, com secas em algumas regiões e inundações em outras (Ropelewski e Halpert, 1986, 1989).
Os Modelos de Circulação Geral Atmosférico (MCGAs) geralmente produzem as previsões climáticas em uma resolução espacial não satisfatória para diversas aplicações, embora maiores resoluções espaciais (menor espaçamento de grade) já sejam possíveis para alguns MCGAs. A aplicação do MCGA em previsão climática (por exemplo, para os próximos 1 a 4 meses) tem sido limitada devido à resolução espacial ser insuficiente para fornecer informações em escala regional com detalhamento adequado (Menéndez et al., 2001; Nicolini et al., 2002; Misra et al., 2003; Qian et al., 2003) especialmente em aplicações que exigem resolução mais refinada como, por exemplo, hidrologia e agricultura. Apesar dos sistemas de grande escala, responsáveis por grande parte da variabilidade da precipitação e temperatura, serem bem resolvidos pelos MCGA, os processos de escala subgrade associados à topografia, cobertura da superfície, processos convectivos úmidos, curvatura da costa dos continentes, que produzem padrões climáticos regionais, não são capturados por estes modelos (Nicolini et al., 2002). Com o objetivo de aprimorar a representação desses processos são utilizados os modelos climáticos regionais (MCR).

Segundo Sen et al. (2004a, 2004b), o interesse nos MCR deve-se à possibilidade de utilizar parametrizações físicas mais apropriadas para resolver processo de mesoescala e também à maior resolução espacial das previsões que estes modelos podem produzir, diminuindo potencialmente os erros encontrados nos MCGA associados à baixa resolução espacial.

Para simular com maior acurácia o clima sazonal, diversos pesquisadores utilizaram a técnica de "downscaling" dinâmico em diversas aplicações (Moncunil et al., 2002; Sun et al., 2005). Esta técnica tem como base o aninhamento de um modelo regional em um MCGA, ou seja, os dados de saída dos MCGA são utilizados como condições iniciais (CI) e de contorno (CC) para os MCR (Dickinson et al., 1989; Giorgi 1990; Liu et al., 1994), tendo como vantagem representar de forma mais detalhada as características regionais de topografia e superfície (Qian et al., 2003; Frías et al., 2005). Vários estudos acerca do uso desta técnica (downscaling) sugerem um melhor desempenho na simulação e previsão de distribuição de precipitação localizada (Nobre et al., 2001; Seth e Rojas, 2003; Sun et al., 2005), embora estudos empregando uma comparação sistemática e compreensiva de previsões climáticas sazonais produzidas por MCGAs e MCRs são raros ou inexistentes.

Portanto, a aplicação da técnica de downscaling (ou redução de escala) torna-se uma ferramenta importante para o usuário poder utilizar a previsão climática em um nível mais compatível com o seu processo de tomada de decisão, dando maiores possibilidades de planejar futuras ações para minimizar os impactos socioeconômicos. Nesta linha de atuação, o objetivo desse estudo foi calibrar e avaliar o modelo regional Brazilian developments on the Regional Atmospheric Modeling System 
(BRAMS, Freitas et al., 2009) aninhado unidirecionalmente ao MCGA do CPTEC (Bonatti, 1996) em prever eventos climáticos extremos sobre a América do Sul, enfocando o trimestre marçoabril-maio (MAM).

\section{MÉTODOS}

\subsection{Descrição dos modelos}

O método que foi usado no presente trabalho consiste na aplicação da técnica de downscaling dinâmico, o qual utiliza o uso de um modelo regional (BRAMS) aninhado unidirecionalmente a um MCGA (no caso, o do CPTEC). Portanto, com a utilização do modelo regional BRAMS para prever as condições climáticas sobre o continente Sul Americano, aumentando-se o detalhamento espacial das previsões, é esperado uma melhoria na qualidade das previsões climáticas regionais sobre a América do Sul. Para avaliar a habilidade deste método em reproduzir os aspectos característicos regionais do clima sobre a América do Sul, o modelo BRAMS foi integrado, de forma retrospectiva, para o período de janeiro a maio de 1983 e 1989.

\subsubsection{MCGA do CPTEC}

O MCGA em uso no CPTEC, para previsão de tempo e estudos climáticos, tem sua origem no Center for Ocean, Land and Atmosphere Studies (COLA) em 1988. Uma versão do modelo global do CPTEC foi colocada em operação em dezembro de 1998, denominada versão 2.0 CPTEC/COLA. Desde então, esse modelo tem sido constantemente atualizado, incluindo novas parametrizações físicas, nova dinâmica e nova estrutura de codificação conforme o padrão Fortran 95, sendo atualmente conhecido como MCGA/CPTEC.

O MCGA que foi utilizado neste trabalho tem a versão descrita em Bonatti (1996), cuja resolução é T062L28, onde T indica o tipo de truncamento espectral utilizado, denominado triangular, na onda zonal de número 62 e L refere-se ao número de camadas na vertical, neste caso, 28. A resolução horizontal do T62 é aproximadamente de $1,875^{\circ} \times 1,875^{\circ}$. Ainda segundo Bonatti (1996), o MCGA do CPTEC resolve as equações primitivas em coordenadas esféricas e na forma espectral. Para facilitar o tratamento espectral, as equações do movimento horizontal são transformadas nas equações da vorticidade e divergência. Portanto, as variáveis previstas pelo MCGA do CPTEC são: logaritmo da pressão à superfície, vorticidade, divergência do vento horizontal, temperatura virtual e umidade específica, além das obtidas a partir das parametrizações físicas (temperatura de superfície, temperatura do interior do solo e do interior do dossel, umidade do solo, chuva, etc.). O MCGA do CPTEC parametriza os processos de escala subgrade como a interação solo-vegetação-atmosfera, camada limite planetária (CLP), transferência radiativa na atmosfera, condensação de grande escala e convecção cumulus profunda e convecção rasa, além da difusão horizontal e local.

Para o presente estudo o estado atmosférico do MCGA foi inicializado com apenas uma condição inicial de reanálise obtida do NCEP/NCAR (Kalnay et al., 1996). A TSM persistida (neste caso, a anomalia de TSM observada do mês de janeiro de 1983 e 1989) foi usada como condição de contorno e a parametrização de convecção utilizada no MCGA/CPTEC foi do tipo Kuo (1974) e Grell (Grell e Dévéndyi, 2002). Uma avaliação da climatologia deste modelo para diversas regiões do globo, assim como, detalhes técnicos adicionais são descritos em Cavalcanti et al. (2002).

\subsubsection{Modelo regional BRAMS}

O Brazilian developments on the Regional Atmospheric Modeling System BRAMS, na versão 5.0 atualmente em uso operacional no CPTEC, foi a ferramenta utilizada nesse estudo para gerar o "downscaling". O BRAMS constitui-se em uma versão brasileira do Regional Atmospheric Modeling System RAMS descrito por Pielke (1984); Pielke et al. (1992) e Walko et al. (1995), o qual foi desenvolvido com o objetivo de criar e aprimorar novos esquemas de parametrizações e atualizações voltadas para a região tropical e subtropical do Brasil.

O RAMS constitui-se num código numérico altamente versátil, podendo ser configurado para fazer simulações da circulação atmosférica em várias escalas de tempo e espaço, bem como, diferentes resoluções horizontais e verticais. O RAMS foi desenvolvido por cientistas da Universidade do Estado do Colorado, nos Estados Unidos da América. Na versão brasileira do RAMS, o BRAMS, há várias inovações e melhorias em termos de física e dinâmica, assim como, qualidade do código e eficiência computacional (Freitas et al., 2009).

$\mathrm{O}$ conjunto de equações dinâmicas e termodinâmicas que governam o movimento atmosférico utilizadas pelo BRAMS possuem as características de ser não-hidrostática e compressível, que consistem em equações prognósticas para as variáveis de estado, componente zonal do vento (u), componente meridional do vento (v), componente vertical do vento (w), temperatura potencial e razão de mistura de água total e hidrometeoros. Esse modelo é baseado em diferenças finitas e usa grade do tipo C de Arakawa (Mesinger e Arakawa, 1976). Neste esquema de grade, as variáveis termodinâmicas e de umidade são definidas no centro dos pontos de grade e suas componentes $\mathrm{u}, \mathrm{v}$, e w são resolvidas nos pontos $\mathrm{dx} / 2$, $\mathrm{dy} / 2$ e dz/2, respectivamente. A coordenada vertical usada pelo modelo é do tipo sigma-z, a qual acompanha o terreno na parte inferior e possui topo plano. Além disto, o BRAMS 
permite o aninhamento múltiplo de grades, o que possibilita que as equações sejam resolvidas de forma mútua nas grades de diferentes resoluções. $\mathrm{O}$ aninhamento de grades proporciona uma alta resolução espacial em áreas selecionadas.

O BRAMS é usualmente aplicado para estudos de mesoescala, com finalidades diversas, que possui várias opções de parametrizações físicas incluídas no seu código. Atualmente, o modelo contém 7 parametrizações para tratamento da difusão turbulenta na camada limite planetária e dentro de nuvens, podendo tratar os coeficientes de difusividade de forma anisotrópica e isotrópica. Para transferência radiativa, 4 submodelos estão disponíveis, sendo um deles com tratamento explícito de água condensada e aerossóis (Longo et al., 2006; Rosário et al., 2012). Microfísica de nuvens que segue o esquema descrito por Walko et al. (1995). Parametrização de convecção rasa é descrita por Souza (1999) e existe uma adicional parametrização introduzida por Freitas et al., (2009) e descrita por Grell e Dévéndyi (2002, daqui em diante GD). Recentemente, foi introduzida no BRAMS uma nova extensão da parametrização GD, chamada G3-D, com novos fechamentos e formulações para o entranhamento lateral. Uma importante característica do esquema GD/G3-D é a possibilidade de usar diferentes fechamentos para o fluxo de massa na base da nuvem, uma combinação de todas através de simples média aritmética ou ainda uma combinação produzida por técnicas em otimização (Santos et al., 2012). Em temos de parametrização este trabalho foi beneficiado com a incorporação do modelo de superfície Joint UK Land Environment Simulaton (JULES) como descrito em Moreira et al. (2013). Recentemente, Grell e Freitas (2014, GF) desenvolveram uma parametrização de convecção adequada para as escalas cinzas e acoplada com aerossóis. Os autores aplicam ideias originalmente desenvolvidas por Arakawa et al. (2011), fazendo com que a parametrização de convecção profunda possa ser aplicada em qualquer escala espacial, incluindo a escala de cinza. Efeitos de aerossóis são contabilizados através da dependência da autoconversão e evaporação com a quantidade de núcleos de condensação na base da nuvem.

Em termos de dinâmica, BRAMS possui agora um aperfeiçoamento no filtro de Asselin (usado no esquema leapfrog de integração temporal) descrito por Williams (2009). Este aperfeiçoamento mantém a ordem de acurácia do esquema de integração que é reduzida para primeira ordem quando se aplica o filtro de Asselin em sua forma original. Aplicação deste filtro foi testado neste estudo para verificar o ganho de qualidade da previsão climática. O esquema de advecção de escalares foi aperfeiçoado com a introdução de um esquema absolutamente monotônico com baixa difusão numérica (Freitas et al., 2012), o que produz melhores padrões de transporte, principalmente em regiões com fortes gradientes, como o caso de frentes frias e presença de nuvens.
A versão 5.0 do BRAMS gera previsões numéricas operacionais no CPTEC/INPE com resolução espacial de 5 $\mathrm{km}$, enquanto que a versão anterior realizava previsões com resolução espacial de $20 \mathrm{~km}$. Para desenvolver a nova versão do BRAMS foi utilizada a formulação não-hidrostática, que representa com maior precisão processos físicos de menor escala, como por exemplo o desenvolvimento e a dissipação de nuvens (chuva). Como mencionado na secção anterior, outros avanços nas parametrizações físicas e na dinâmica do modelo também foram realizados, como por exemplo, a parametrização de convecção GD/G3-D e GF.

\subsection{Configuração do experimento}

As condições iniciais e de contorno das variáveis atmosféricas prognósticas de grande escala usadas para integrar o BRAMS foram obtidas do MCGA do CPTEC, para os meses de janeiro a maio de cada ano do período 1983 e 1989, sendo o foco deste trabalho o trimestre MAM, que representa o extremo da estação chuvosa da região Nordeste do Brasil (NEB). Os fenômenos climáticos (El Niño e Lã Niña) causam transtornos à sociedade e influenciam diretamente o regime pluviométrico do Norte, setor norte do Nordeste e Sul do Brasil. As variáveis previstas pelo MCGA do CPTEC, ou seja, altura geopotencial, temperatura do ar, componentes meridional e zonal do vento e umidade relativa foram utilizadas para iniciar o modelo regional BRAMS, e também como condições de contorno com atualização a cada 6 horas. A resolução horizontal do MCGA foi de $1,875^{\circ} \times 1,875^{\circ}(\sim 200 \mathrm{~km})$ de latitude e longitude. A condição de contorno do modelo é informada a cada passo no tempo usando o esquema de Relaxação Newtoniana (ou "nudging") sendo aplicada num conjunto lateral de pontos de grade da borda e no topo do modelo. As escalas temporais de relaxação são informadas adiante.

Para o modo de previsão climática sazonal, a condição de contorno oceânica foi a TSM persistida. A anomalia de TSM observada do primeiro mês da previsão (janeiro) é persistida nos 4 meses seguintes, essa mesma condição foi usada nas previsões do MCGA. No procedimento de persistência, a anomalia de TSM do mês de janeiro foi somada às climatologias (1982-2006) de TSM de cada um dos meses de fevereiro a maio. Para confecção das anomalias e climatologia da TSM foram utilizados os dados produzidos por Reynolds et al. (2002), espaçados em uma grade de $1^{\circ} \times 1^{\circ}$ de latitude e longitude. A umidade do solo foi a climatologia de um período de 6 anos (1997-2002), proveniente das estimativas de umidade (Gevaerd e Freitas, 2006) obtidas a partir dos dados de precipitação do Global Precipitation Climatology Project (GPCP, http://precip.gsfc.nasa.gov/gpcp daily_comb.html), com resolução espacial de $1^{\circ} \times 1^{\circ}$ de latitude e longitude e resolução temporal diária (Huffman et al., 2001). 
O espaçamento de grade do BRAMS foi de $30 \mathrm{~km}$, centrada na latitude de $19^{\circ} \mathrm{S}$ e na longitude de $54,4^{\circ} \mathrm{W}$ e 42 níveis verticais, e o domínio compreende toda a AS e parte dos Oceanos Atlântico e Pacífico $\left(60^{\circ} \mathrm{S}-20^{\circ} \mathrm{N}\right.$ e $\left.110^{\circ} \mathrm{W}-0^{\circ}\right)$, Figura 1. É ideal que o domínio seja suficientemente grande para que o modelo possa desenvolver sua própria circulação regional (Giorgi e Mearns, 1999; Chou et al., 2005). As previsões do modelo regional, para o trimestre MAM dos anos de 1983 e 1989, foram iniciadas às 1200 UTC do dia 13 de janeiro e se estendem até o último dia do quinto mês. Os primeiros 18 dias de previsão foram considerados como de "spin-up" e excluídos das avaliações, sendo que para esses dias foi usada a condição de contorno oceânica (TSM) observada, esse é um procedimento comumente usado na elaboração de previsões climáticas sazonais no CPTEC.

As parametrizações adotadas no experimento são: para transferência radiativa CARMA (descrita em Longo et al., 2006); no processo de convecção profunda a parametrização é do tipo GF (Grell e Freitas, 2014), com fechamento ensemble. A parametrização de convecção rasa é descrita por Souza (1999); para a difusão turbulenta o fechamento é de ordem 2.5 (Mellor e Yamada, 1982); a microfísica de nuvens segue o esquema descrito por Walko et al. (1995), com prognóstico de 7 hidrometeoros. A escala do TNUDLAT foi de 3600 segundos e do TNUDTOP de 10800 segundos.

\subsection{Dados}

Os dados usados para validar o padrão da precipitação sazonal prevista pelo modelo numérico foram dados de precipitação do Climate Prediction Center (CPC), descritos em Chen e Xie (2008), com resolução horizontal de $0.5^{\circ} \times 0.5^{\circ}$ de latitude e longitude. O CPC é considerado um conjunto

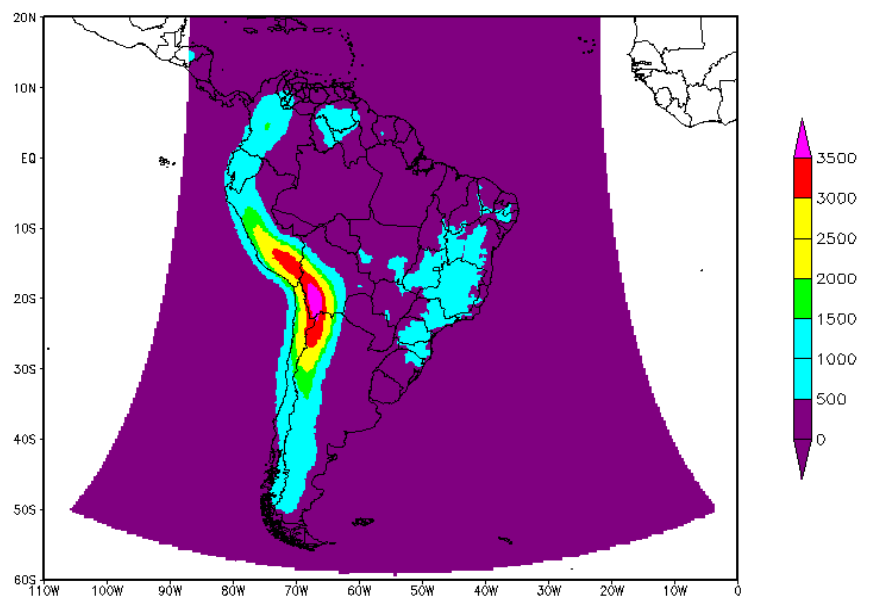

Figura 1 - Mapa do domínio do modelo e topografia (m) da América do Sul. de dados de referência e já foi avaliado para estudos de modelagem climática (Silva et al., 2007). Estes dados são baseados principalmente em dados diários de chuva observada, interpolados pelo método de interpolação ótima (Gandin, 1965).

\subsection{Metodologia para avaliação das previsões}

No presente trabalho foram realizadas integrações para 1983 e 1989 com o BRAMS aninhado unidirecionalmente ao MCGA do CPTEC. Neste trabalho apenas a variável precipitação acumulada foi avaliada. As previsões foram avaliadas de forma subjetiva para identificar se o modelo consegue capturar o padrão observado. O desempenho das previsões do modelo regional foi investigado em todo o domínio da América do Sul.

\section{RESULTADOS E DISCUSSÕES}

Visto a importância da qualidade e confiabilidade das previsões climáticas sazonais no âmbito sócio-econômico, vislumbrou-se a possibilidade de poder contribuir com mais uma ferramenta numérica para este prognóstico. Portanto, nesta seção são apresentadas informações sobre os procedimentos de calibração do modelo BRAMS para a escala de clima sazonal. Alguns testes de parametrizações físicas (tais como convecção, radiação e superfície) e influência da condição inicial foram realizados a fim de se obter uma versão a qual pudesse representar o clima de forma mais realística possível, na presente versão do modelo. Todos os testes foram realizados para dois episódios de extremos climáticos um ano de El Niño (1983) e um ano de La Niña (1989), durante o trimestre MAM que é o foco deste trabalho. Ambos os episódios foram classificados como fortes (www.cptec.inpe.br/enos).

Diversos processos físicos não são resolvidos explicitamente (isto é, na escala de grade computacional) pelos modelos e precisam ser parametrizados, ou seja, seus efeitos sobre a escala de grade devem ser representados estatisticamente. Os modelos necessitam de diferentes níveis de complexidade física que estão relacionados com as escalas temporais envolvidas como por exemplo: tempo, clima ou projeções climáticas, bem como as escalas espaciais utilizadas.

Dentre os processos físicos (radiação, turbulência, nuvens, superfície e outros) mais importantes para a previsão do clima destacam-se a radiação solar incidente, a mudança na cobertura vegetal, os fluxos de superfície, corpos d'água, TSM e entre outros (Moncunil et al., 2002). Os autores encontraram necessidades de ajustes nas parametrizações utilizadas no BRAMS 5.0 para sua versão climática. Desta forma, vários testes foram feitos até obter uma versão que pudesse representar de forma satisfatória a evolução climática da atmosfera 
desenvolvida pelo modelo BRAMS. Para reduzir o volume de processamento computacional neste trabalho, apenas um membro (CI do dia 13 de janeiro) foi utilizado para simular e prever os anos estudados. Entretanto, após realizar a calibração do modelo, para a avaliação histórica da destreza das previsões do modelo calibrado para o período de 1979-2010, foi gerado um conjunto de 10 membros de previsões para cada ano, cujos resultados serão apresentados em um próximo trabalho. Dessa forma, será possível realizar uma avaliação das incertezas associadas às previsões climáticas produzidas.

Na Figura 2 são apresentados os mapas da climatologia de precipitação observada (1979-2010) e da precipitação acumulada nos anos de 1983 e 1989, para o trimestre MAM. Climatologicamente (Figura 2a), este trimestre caracteriza-se pelos elevados totais pluviométricos, em torno de $1600 \mathrm{~mm}$ sobre a região Norte e setor norte da região Nordeste devido ao deslocamento anual da ZCIT. Sobre a região Sul do Brasil e na costa leste do NEB, são observados totais da ordem de 400 $\mathrm{mm}$, em razão da circulação de brisa que advecta umidade para o continente. No interior do NEB são observados reduzidos volumes de precipitação caracterizando o clima semiárido dessa região, com acumulados de precipitação inferior a 400 $\mathrm{mm}$. O extremo norte da AS também apresenta déficit de precipitação. As regiões Sudeste e Centro-Oeste do Brasil, Bolívia e Paraguai apresentam uma diminuição gradativa da precipitação representando o término da sua estação chuvosa compreendida entre os meses de dezembro a fevereiro. A precipitação nessa região, durante o trimestre MAM, deve-se

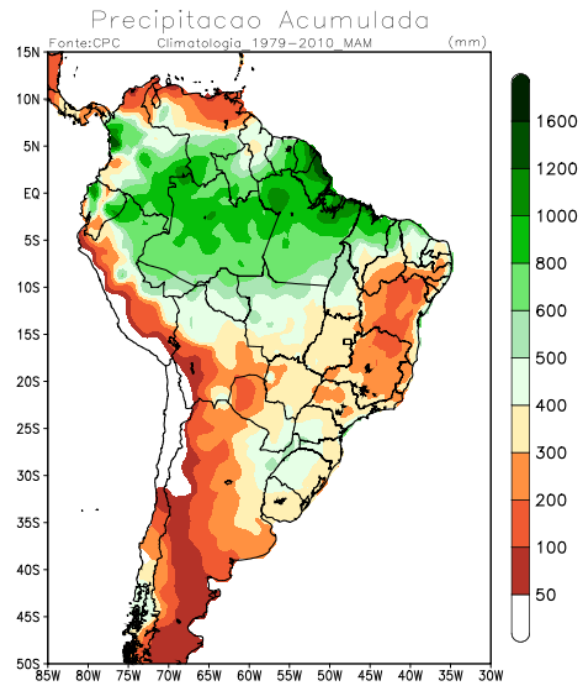

(a)

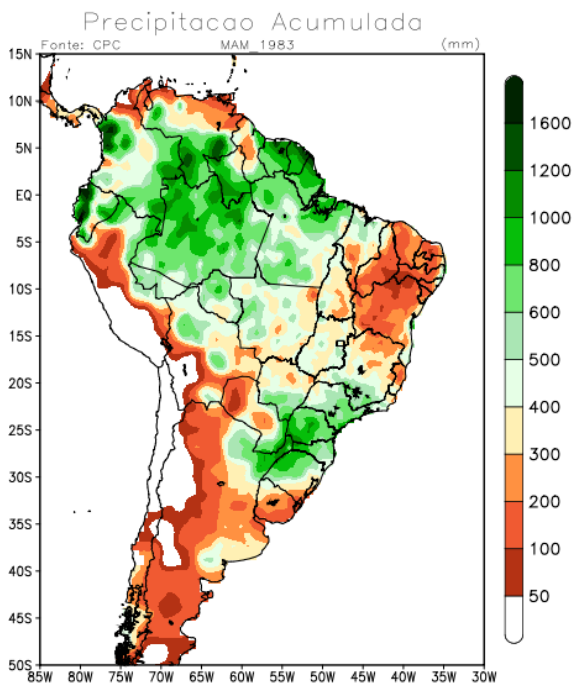

(b)

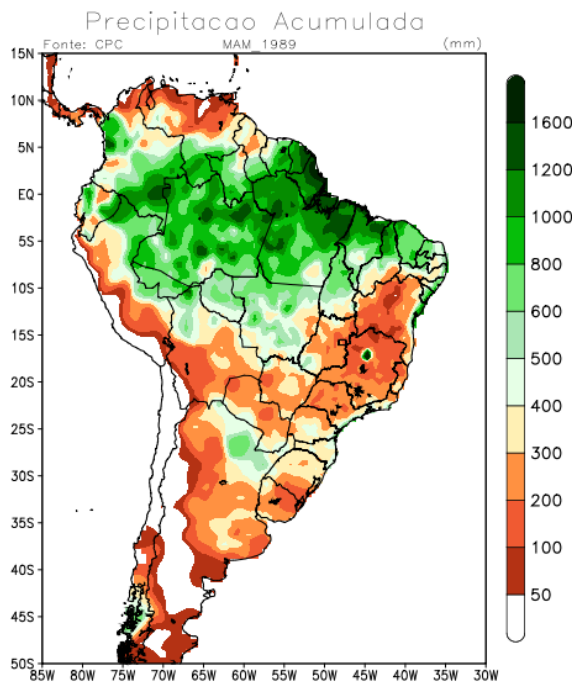

(c)

Figura 2 - Mapas da precipitação acumulada (mm) durante o trimestre MAM para média climatológica (a), El Niño de 1983 (b) e La Niña de 1989 (c). Os dados de precipitação foram fornecidos pelo CPC (CHEN; XIE, 2008). 
principalmente a sistemas sinóticos oriundos da região Sul da AS, que conseguem atingir latitudes mais ao Norte, com algumas diferenças na intensidade dos sistemas. A região Sul do Brasil, Uruguai e norte/nordeste da Argentina possui um regime de chuva espacialmente uniforme durante MAM, verifica-se acumulados da ordem de até $600 \mathrm{~mm}$, sendo a maior parte dessa precipitação associada à passagem de sistemas frontais.

A Figura 2 b mostra o padrão da distribuição de precipitação sobre a AS durante um evento de El Niño. Nessa figura observa-se precipitação reduzida no setor norte e leste da Amazônia e norte do NEB e aumento da precipitação na costa oeste da AS, região Sul do Brasil e setor nordeste da Argentina. Para o ano de Lã Niña (Figura 2c) verifica-se redução de precipitação sobre grande parte da região Sul do Brasil e aumento de precipitação principalmente no Norte e setor norte do NEB.

Nas previsões e simulações com o modelo BRAMS, para fins de calibração, foi utilizado o mesmo código fonte usado para gerar as previsões de tempo operacional do CPTEC/ INPE. Porém, algumas alterações foram feitas na tentativa de adequar as previsões para o modo climático. A grade do modelo foi alterada de $5 \mathrm{~km}$ para $30 \mathrm{~km}$, devido, principalmente ao caráter climático da simulação não exigir, a princípio, esse detalhamento de grade $(5 \mathrm{~km})$ e as limitações computacionais. A parametrização de convecção profunda foi alterada de GD/ G3-D para GF, que foi recentemente desenvolvida para escalas de 1 a $100 \mathrm{~km}$.

A Figura 3 mostra os mapas de precipitação acumulada (mm) para os eventos de El Niño de 1983 e La Niña de 1989, previstos pelo modelo BRAMS. Observa-se que o modelo

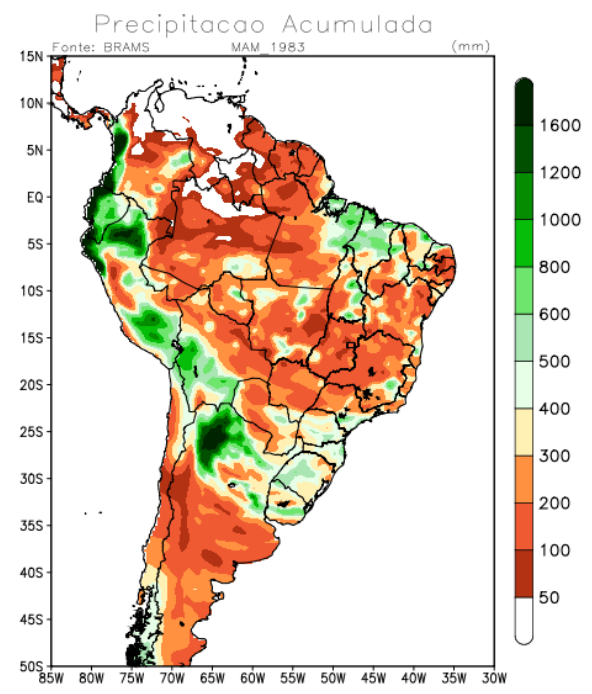

(a) subestima a precipitação observada (Figuras 2b e 2c) em praticamente todo o território brasileiro. Na costa oeste da AS, entre a Colômbia e extremo norte do Chile, o modelo BRAMS superestima a observação. Desta forma, o resultado da previsão não foi satisfatório ao utilizar o código fonte da previsão de tempo apenas com as modificações acima descritas. Assim, observou-se a necessidade de investigar onde o modelo estava divergindo seus resultados com os dados observados, para tentar calibrar as parametrizações físicas e melhorar seu desempenho. E, com isso, poder inferir sobre a capacidade do modelo regional BRAMS em realizar previsões de clima.

Partindo do código que gerou os resultados da Figura 3, foram feitos testes modificando a configuração física do modelo BRAMS, para tentar entender o porquê da ausência de precipitação sobre a AS nas simulações feitas pelo modelo. A primeira mudança foi feita na parametrização de radiação, onde foi introduzida uma fração de cobertura de nuvens da ordem de $20 \%$. Na versão anterior era considerada uma fração de cobertura de até $100 \%$. Na forma anterior, o modelo apresentava um déficit de radiação solar em superfície produzindo uma atmosfera excessivamente fria e estável em baixos níveis, com forte impacto no balanço de radiação. Visto que, a radiação solar é um dos principais processos físicos quando se trata de previsão de clima, esta precisa ser bem resolvida pelo modelo a fim de contribuir com maior acurácia na representação das condições climáticas observadas. Outras configurações também foram adotadas, o coeficiente de peso do nudging (relaxação Newtoniana), para temperatura e umidade, foi reduzido de 1 para 0.6 , deixando o modelo mais livre para calcular/prognosticar essas variáveis, do que em relação ao vento horizontal. No caso

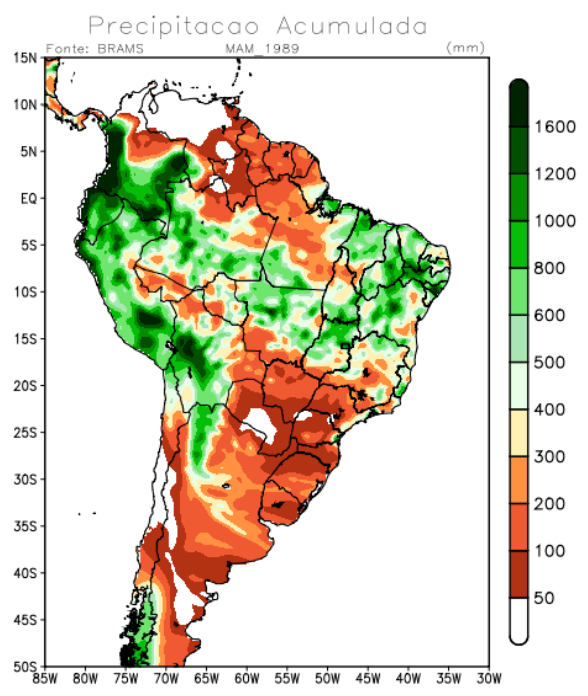

(b)

Figura 3 - Mapas da precipitação acumulada (mm) durante o trimestre MAM para o El Niño de 1983 (a) e La Niña de 1989 (b) prevista pelo modelo BRAMS. 
da pressão, o valor foi alterado de 1 para 4 impondo ao modelo maior consistência neste parâmetro com as análises usadas como condição inicial. A turbulência escolhida nesta previsão foi a originalmente desenvolvida por Mellor e Yamada (1974), e não foi usada a opção de advecção monotônica, custosa do ponto de vista computacional para a escala de previsão aqui utilizada (os resultados de sensibilidade não mostraram ganhos significativos nesta escala espacial). E por fim, utilizou-se uma parametrização convectiva recém desenvolvida que apresenta um comportamento suave na transição de escalas, incluindo a escala cinza da convecção profunda. Esta ainda inclui efeitos de aerossóis que são contabilizados através da dependência da autoconversão e evaporação com a quantidade de núcleos de condensação na base da nuvem, apesar desta opção não ser aplicada no presente estudo.

Os mapas da Figura 4 mostram a precipitação acumulada (mm) utilizando uma versão calibrada do modelo BRAMS para previsão climática sazonal, cujas modificações foram citadas no parágrafo anterior. Para o ano de El Niño (Figura 4a) é possível observar que o modelo consegue capturar o padrão de precipitação associada a esse evento, como a redução da precipitação, principalmente no leste da região Norte e NEB, e o aumento de precipitação na região Sul. Na Figura 4b, um padrão de redução de precipitação sobre a região Sul da AS e aumento sobre maior parte da região Norte da AS foi verificado durante o evento de La Niña. Em ambos os anos o modelo tende a subestimar a precipitação nas regiões Norte, principalmente na Amazônia, e Sul do Brasil e a superestimar em praticamente toda a região Nordeste. Esses resultados concordam com os obtidos por Seth e Rojas (2003). O MCGA do CPTEC (CI do

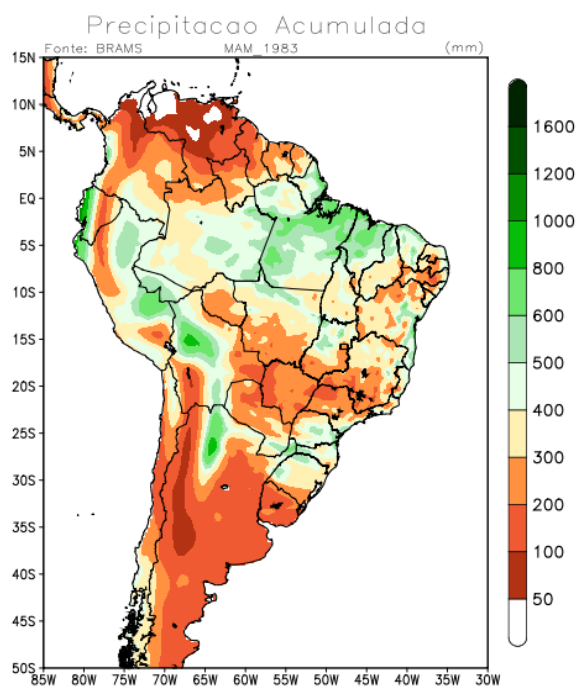

(a) modelo regional) apresenta uma superestimativa de precipitação sobre os Andes, esse comportamento pode estar relacionado a erros numéricos na representação da topografia desta região (Figueroa, 1997) que por sua vez vai ocasionar erros nos processos convectivos (Oyama, 2006). Esse erro segue para o modelo regional, que também tem dificuldade em representar a topografia sobre os Andes.

Até o momento os mapas de precipitação da Figura 4 são resultados do modelo regional no modo de previsão, onde, as CI são as previsões do MCGA do CPTEC e a CC a TSM persistida. Para tentar estudar com mais detalhes os processos físicos inseridos no BRAMS foi feita uma simulação, com CI (reanálises do National Center for Enviromental Prediction NCEP) e como CC a TSM observada para o período de estudo. As CI e de fronteira fornecem informações sobre a estrutura atmosférica, que orientam os sistemas sinóticos e após essa fase o modelo simula as circulações geradas pelas forçantes locais (topografia) e/ou embebidas nos sistemas de grande escala. Portanto, e em princípio, quanto maior a qualidade dos dados iniciais e de contorno melhor será o resultado da simulação.

A finalidade dessa simulação é verificar a habilidade do modelo em reproduzir informações climáticas em alta resolução baseada apenas na sua física interna. E, diante dos resultados encontrados poder sugerir novas mudanças na configuração do modelo, visando gerar um produto com maior destreza e confiabilidade, principalmente para os processos de tomada de decisão.

A Figura 5 mostra os mapas de precipitação acumulada (mm) utilizando a CI e CC observadas para ambos os eventos climáticos extremos. Observa-se que a simulação (Figura 5a)

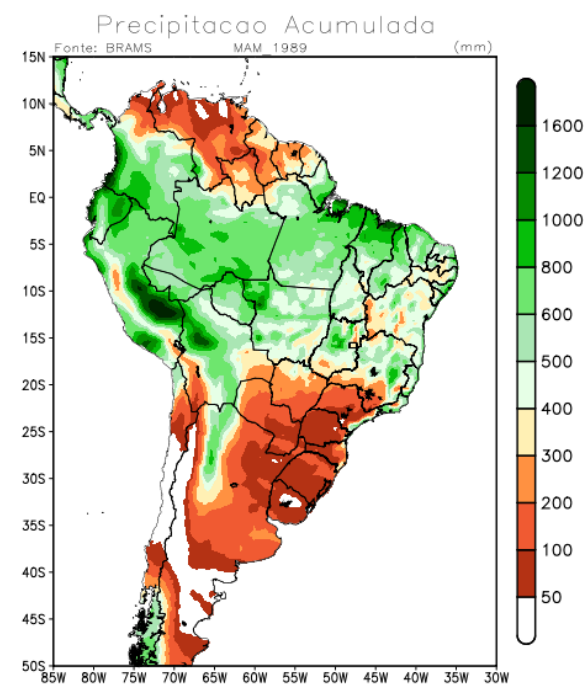

(b)

Figura 4 - Mapas da precipitação acumulada (mm) durante o trimestre MAM para o El Niño de 1983 (a) e La Niña de 1989 (b) prevista pelo modelo BRAMS modificado. 
apresentou melhores resultados sobre a região Norte da AS, principalmente sobre os Estados do Amazonas e Mato Grosso, quando comparado com os resultados da previsão do modelo regional (Figura 4a). Para as regiões Nordeste e Sul, o inverso foi verificado, a previsão do modelo regional para o ano de El Niño (Figura 4a) foi mais coerente com a observação (Figura 2b) do que a simulação (Figura 5a). Para o ano de 1989 a simulação do modelo regional (Figura 5b) esteve mais próxima da observação (Figura 2c) do que a previsão do modelo (Figura 4b). Para ambos os anos, tanto a previsão do modelo como a simulação, o modelo superestimou a precipitação sobre os Andes.

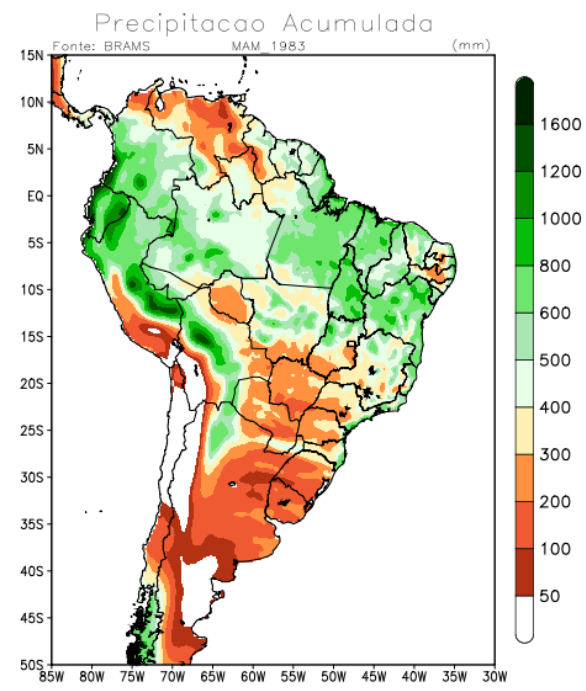

(a)
Partindo dos resultados apresentados na Figura 5, observou-se a necessidade de estudar o impacto da CI do MCGA/CPTEC, produzidas com diferentes representações de parametrizações de convecção profunda nas previsões climáticas de eventos extremos com o BRAMS. As previsões do modelo global (Figura 6), que até o momento alimentaram o modelo regional, utilizaram como parametrização de convecção profunda o esquema de Kuo (1974). Este esquema baseia-se fundamentalmente na convergência de umidade de grande escala, porém, tem como desvantagem a não representação das correntes descendentes em escala convectiva e processos

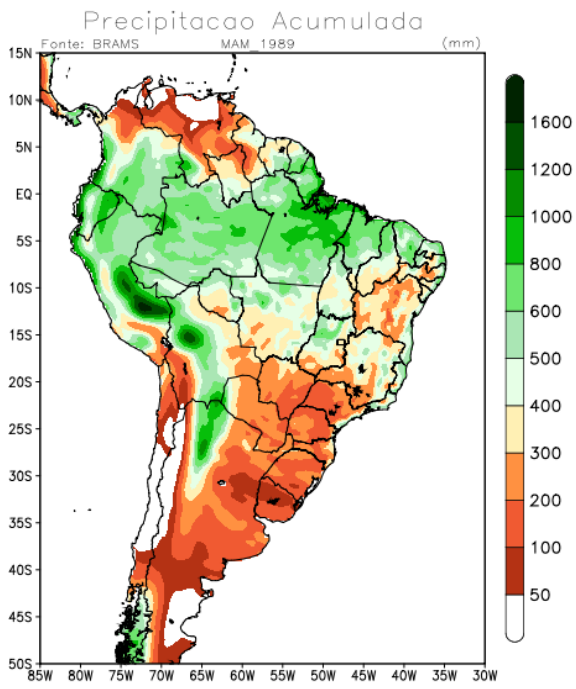

(b)

Figura 5 - Mapas da precipitação acumulada (mm) durante o trimestre MAM para o El Niño de 1983 (a) e La Niña de 1989 (b) simulada pelo modelo BRAMS modificado e com condição de contorno observada.

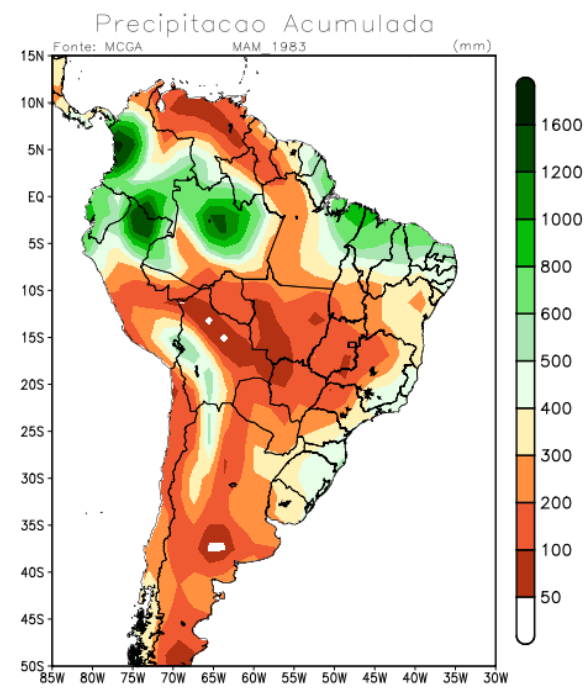

(a)

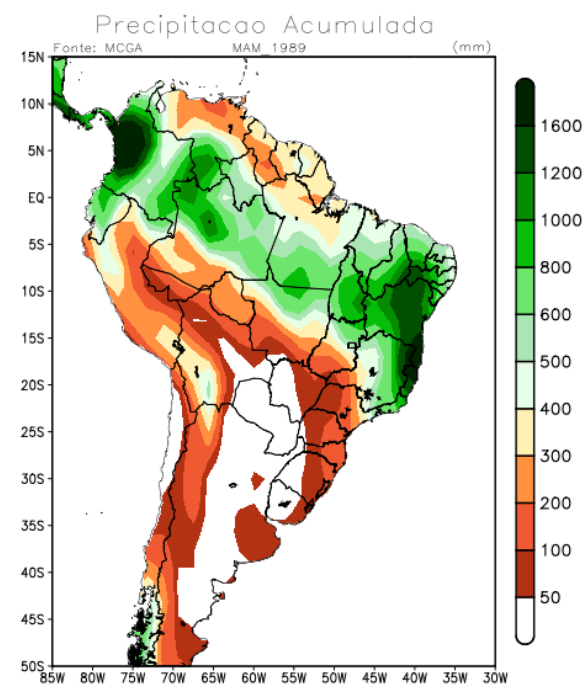

(b)

Figura 6 - Mapas da precipitação acumulada (mm) durante o trimestre MAM para o El Niño de 1983 (a) e La Niña de 1989 (b) prevista pelo modelo MCGA/CPTEC com parametrização de convecção do tipo KUO. 
de mesoescala. O MCGA também foi rodado como a parametrização do tipo Grell (Grell e Dévéndyi, 2002) para gerar CI para o modelo regional (Figura 8). O esquema Grell inclui efeitos de correntes descendentes e se baseia em somente um tipo de nuvem com entranhamento pré-estabelecido. O fechamento para definição da intensidade do processo convectivo (em termo de fluxo de massa na base da nuvem) se baseia na hipótese de quase-equilíbrio (Arakawa e Schubert, 1974). Os testes de parametrização de convecção profunda no MCGA/CPTEC foram realizados para tentar identificar qual

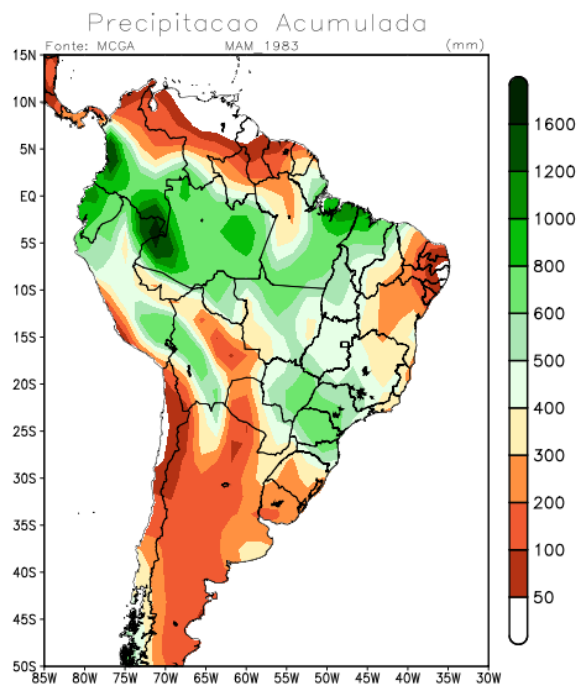

(a) o tipo de parametrização apresenta melhor resultado para as previsões climáticas sobre a AS.

As Figuras 6a e 7a mostram os mapas de precipitação acumulada (mm) para o ano de 1983 (El Niño) prevista pelo MCGA do CPTEC, com parametrização do tipo Kuo e Grell, respectivamente. É possível notar que o modelo global, sobre influência da parametrização do tipo Grell (Figura 7a), apresenta melhores resultados em toda a AS, exceto na região central do Brasil, onde superestima os acumulados de precipitação observados (Figura 2a). Para o ano de La Niña

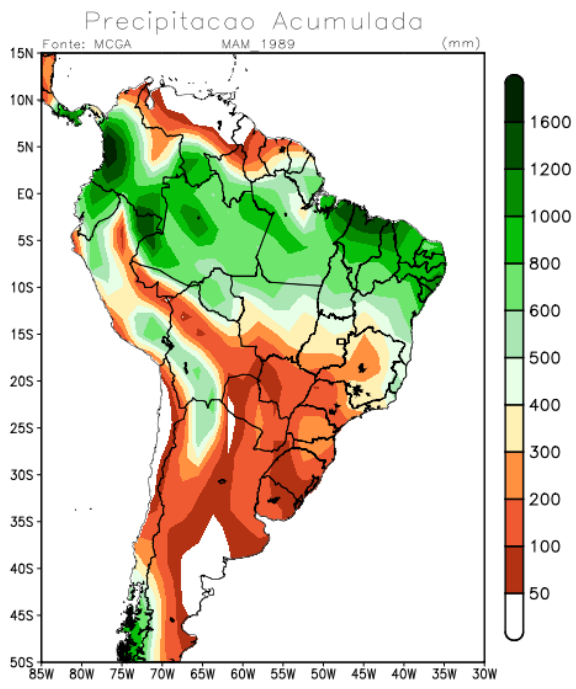

(b)

Figura 7 - Mapas da precipitação acumulada (mm) durante o trimestre MAM para o El Niño de 1983 (a) e La Niña de 1989 (b) prevista pelo modelo MCGA/CPTEC com parametrização de convecção do tipo GRELL.

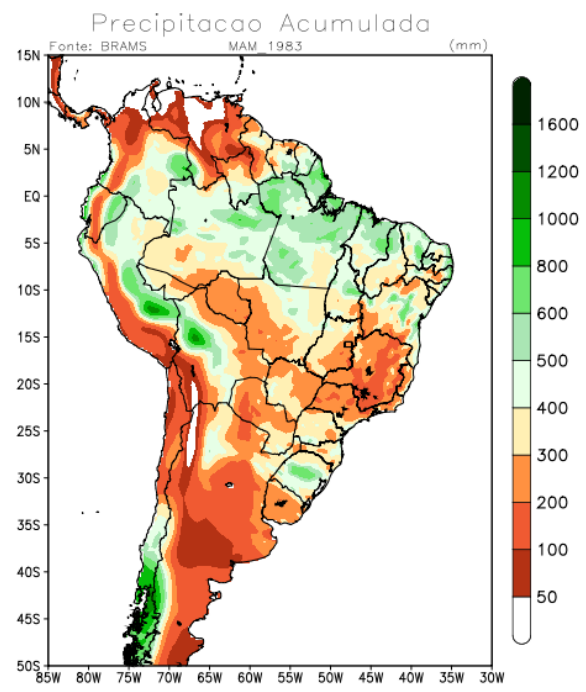

(a)

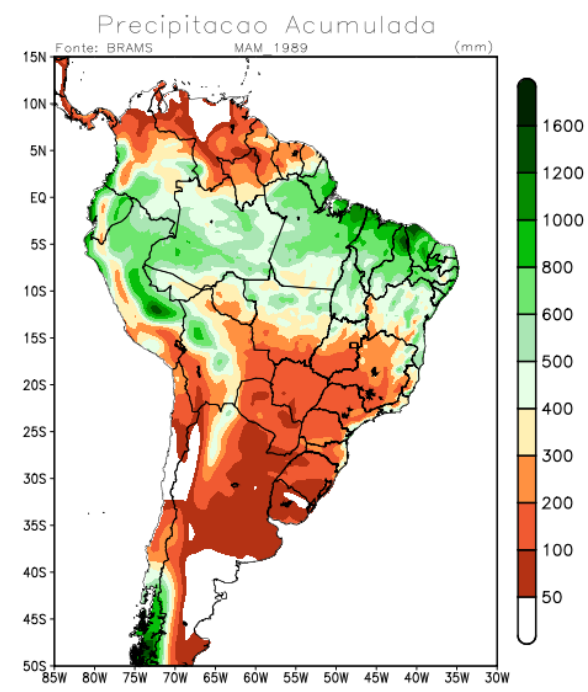

(b)

Figura 8 - Mapas da precipitação acumulada (mm) durante o trimestre MAM para o El Niño de 1983 (a) e La Niña de 1989 (b) prevista pelo modelo BRAMS modificado com CI do MCGA com convecção do tipo GRELL. 
(Figuras $6 \mathrm{~b}$ e 7b), a previsão do modelo global do CPTEC também apresenta melhores resultados com a parametrização do tipo Grell, do que com a parametrização do tipo Kuo. No entanto, ambas as parametrizações de convecção profunda (Figuras 6b e 7b) subestimam a precipitação observada (Figura 2c) nas regiões Sul e Sudeste do Brasil e sobre a Argentina. Para os eventos aqui estudados a previsão do modelo global com parametrização do tipo Grell representa melhor as condições climáticas observadas.

Comparando os resultados do modelo regional, sobre a influência da CI do MCGA com diferentes parametrizações de convecção (Figuras 4 e 8 ), com os mapas de precipitação observada (Figura 2) verifica-se que para o ano de El Niño (1983) a previsão, utilizando o esquema Grell, apresenta resultados razoavelmente melhores no setor norte e leste da Amazônia. $\mathrm{Na}$ região Sul, a previsão utilizando a CI com esquema Kuo apresentou resultados mais satisfatórios. Para o ano de La Niña (1989) o esquema Kuo apresentou melhores resultados sobre toda região Norte da AS e setor Sul do NEB. Sobre a região Sudeste o esquema Grell apresentou resultados mais próximos dos observados (Figura 2b). Na região Sul do Brasil ambos os esquemas subestimaram a precipitação observada.

\section{CONCLUSÕES}

Este trabalho representa uma contribuição aos estudos de modelagem climática sazonal com ênfase na estação chuvosa na região Nordeste do Brasil. O objetivo principal foi calibrar e avaliar o modelo regional BRAMS, aninhado unidirecionalmente ao modelo global MCGA/CPTEC, em prever os eventos climáticos extremos de 1983 (El Niño) e 1989 (La Niña) sobre a AS, para o trimestre MAM. As previsões com o BRAMS foram produzidas na resolução espacial de $30 \mathrm{~km}$, tendo como condições de contorno a temperatura da superfície do mar persistida e a umidade do solo climatológica. Uma das potenciais vantagens da técnica de aninhamento unidirecional (downscaling) é o melhor detalhamento espacial das previsões, reduzindo a resolução horizontal de $\sim 200 \mathrm{~km}$ do modelo global (MCGA/CPTEC) para $\sim 30 \mathrm{~km}$ do modelo regional (BRAMS). Adicionalmente, através dessa técnica os modelos regionais tem potencial de resolver melhor os processos físicos de sub-grade que não são tratados pelo modelo global.

Quando inseridas as mudanças físicas na radiação solar (um dos principais processos físicos quando se trata de previsão de clima), convecção profunda e influência da condição inicial feitos no modelo BRAMS, o modelo consegue capturar os padrões de precipitação associada os dois eventos climáticos extremos investigados, como a redução (aumento) da precipitação, principalmente no leste da região Norte e NEB, e o aumento (redução) de precipitação na região Sul para o ano e
El Niño (La Niña), porém algumas deficiências foram notadas. Na maior parte da AS, por exemplo, a precipitação do modelo global com o esquema de convecção Grell se aproxima mais da observação do que com o esquema Kuo. Na região Norte do Brasil, principalmente no estado do Amazonas, setor sul do NEB e região Sul do Brasil, o modelo global com o esquema Grell tem melhor desempenho do que o modelo regional, sendo esta uma deficiência do modelo regional que necessita ser corrigida em trabalhos futuros.

Apesar dos diversos avanços no modelo BRAMS descritos neste trabalho, vários processos ainda requerem melhoria em suas representações físicas para um aprimoramento do sistema regional de previsões climáticas sazonais aqui proposto. Por exemplo, os esquemas de radiação e convecção profunda necessitam serem revistos mais detalhadamente, incluindo seus acoplamentos com a microfísica de nuvens. Este é um trabalho contínuo que vem sendo realizado pela comunidade científica do BRAMS. Os autores também reconhecem que outro ponto importante, referente a dinâmica do modelo, também necessita de aprimoramentos. Métodos de alta ordem de integração espacial e temporal, e mais conservativos estão sendo introduzidos em novas versões do modelo e deverão produzir importante ganho de desempenho, principalmente em integrações de longo prazo, como as tratadas neste artigo. Trabalhos futuros incluirão também uma nova coordenada vertical, shaved-eta, que é indicada para situações de existência de complexos topográficos como a cadeia dos Andes. Por fim, planeja-se realizar investigações com outros conjuntos de condições iniciais atmosféricas, umidade do solo e condição de contorno oceânica, com o objetivo de melhorar a inicialização do modelo.

\section{AGRADECIMENTOS}

Os autores agradecem o suporte financeiro da Coordenação de Aperfeiçoamento de Pessoal de Nível Superior (CAPES), pela concessão da bolsa de mestrado do primeiro autor. CASC foi apoiado pelo Conselho Nacional de Desenvolvimento Científico e Tecnológico (CNPq), processo 306863/2013-8. Os autores agradecem os comentários e sugestões de dois revisores anônimos que contribuíram para melhorar a qualidade do trabalho.

\section{REFERÊNCIAS BIBLIOGRÁFICAS}

ARAKAWA, A., JUNG, J.-H.;WU, C.-M.: Toward unification of the multiscale modeling of the atmosphere. Atmospheric Chemistry Physics, 11, 3731, 2011. ARAKAWA, A.; SCHUBERT, W. H. Interaction of a Cumulus Cloud Ensemble with the Large-Scale Environment, Part I. Journal of Atmospheric Sciences ,31, 674-701,1974. 
BONATTI, J. P. Modelo de circulação geral atmosférico do CPTEC. Climanálise Especial, edição comemorativa de, v. 10, 1996. Disponível em: <http://climanalise.cptec.inpe. br/ rclimanl/boletim/cliesp10a/>. Acesso em: 20 setembro 2013.

CAVALCANTI, I. F. A. et al. Global climatological features in a simulation using the CPTEC-COLA AGCM. Journal of Climate, v. 15, n. 21, p. 2965-2988, 2002.

CHEN, M.; XIE, P. CPC precipitation working group. CPC Unified Gauge-based Analysis of Global Daily Precipitation, Western Pacific Geophysics Meeting, Cairns, Australia, 2008.

CHOU, S. C.; BUSTAMANTE, J. F.; GOMES, J. L. Evaluation of Eta model seasonal precipitation forecasts over South America. Nonlinear Processes in Geophysics, v. 12, n. 4, p. 537-555, 2005.

DICKINSON, R. E.; ERRICO, R. M.; GIORGI, F.; BATES, G. T.A regional climate model for the western United States. Climatic Change, v. 15, n. 3, p. 383-422, 1989.

FIGUEROA, S. N. Estudo dos sistemas de circulação de verão sobre a América do sul e suas simulações com modelos numéricos. 1997-08 181 p. (INPE-7121-TDI/672). Tese (Doutorado em Meteorologia) - Instituto Nacional de Pesquisas Espaciais, Sao Jose dos Campos, 1997. Disponivel em: http://urlib.net/sid.inpe.br/deise/1999/06.15.09.40 >. Acesso em: 21 maio 2014.

FREITAS, S. R.; LONGO, K. M.; SILVA DIAS, M. A. F. et al. The Coupled Aerosol and Tracer Transport model to the Brazilian developments on the Regional Atmospheric Modeling System (CATT-BRAMS) - Part 1: Model description and evaluation. Atmospheric Chemistry Physics, v. 9, p. 2843-2861, 2009.

FREITAS, S. R.;RODRIGUES, L.F.;LONGO, K.M.; PANETTA, J. Impact of a monotonic advection scheme with low numerical diffusion on transport modeling of emissions from biomass burning. Journal of Advances in Modeling Earth Systems, v. 4, p. M01001, 2012.

FRÍAS, M. D.; FERNÁNDEZ, J.; SÁENZ, J.; PUEBLA, C.-R. Operational predictability of monthly average maximum temperature over the Iberiam Peninsula using DEMETER simulation and downscaling. Tellus,v. 57, p.448-463, 2005.

GANDIN, L. S. Objective analysis of meteorological fields. Jerusalem: Israel program for scientific translations, 1965.

GEVAERD, R.; FREITAS, S. R. Estimativa operacional da umidade do solo para iniciação de modelos de previsão numérica da atmosfera parte i: descrição da metodologia e validação. Revista Brasileira de Meteorologia, v.21, n.3, p. 1-15, 2006.

GIORGI, F. Simulation of regional climate using a limited area model nested in a general circulation model. Journal of Climate, v. 3, n. 9, p. 941-963, 1990.
GIORGI, F.; MEARNS, L. O. Introduction to special section: Regional climate modeling revisited. Journal of Geophysical Research: Atmospheres, v. 104, n. D6, p. 6335-6352, 1999.

GODDARD, L.; MASON, S. J.;ZEBIAK, S. E.; ROPELEWSKI, C. F.; BASHER, R.; CANE, M. A. Current approaches to seasonal-to-interanual climate predictions. International Journal of Climatology, v. 21, p. 1111-1152, 2001.

GRELL, G.A.; DÉVÉNYI, D.A new approach to parameterizing convection using ensemble and data assimilation techniques. Geophysical Research Letters.v.29, p.1693, 2002.

GRELL, G. A.; FREITAS, S. R. A scale and aerosol aware stochastic convective parameterization for weather and air quality modeling. Atmospheric Chemistry Physics, 14, 5233-5250, 2014.

HUFFMAN, G.J. et al. Global precipitation at one degree daily resolution from multi satellite observations. Journal of Hydrometeorology, v. 2, p. 36-50, 2001.

KALNAY, E. et al. The NCEP/NCAR 40-year Reanalysis Project. Bulletin of the American Meteorological Society, v. 77, n. 3, p. 437-471, 1996.

KUO, H. L. Further studies of the parameterization of the influence of cumulus convection on large-scale flow. Journal of Atmospheric Sciences, v. 31, p.1232-1240, 1974.

LIU, Y.; GIORGI, F.; WASHINGTON, W. M. Simulation of summer monsoon climate over east Asia with an NCAR Regional Climate Model. Monthly Weather Review, v. 122, n. 10, p. 2331-2348, 1994.

LONGO, K. M.; FREITAS, S. R.; DIAS, M. A. S.; DIAS, P. L.S. Numerical modeling of the biomass-burning aerosol direct radiative effects on the thermodynamics structure of the atmosphere and convective precipitation. In: International Conference on Southern Hemisphere Meteorology and Oceanography (ICSHMO), p. 283-289, 2006.

MELLOR, G. L.; YAMADA, T. A Hierarchy of Turbulence Closure Models for Planetary Boundary Layers. Journal of Atmospheric Sciences, 31, 1791-1806, 1974.

MELLOR, G. L.; YAMADA, T. Development of a turbulence closure model geophysical fluid problems. Reviews of Geophysics and Space Physics, v.20, p.851-857, 1982.

MESINGER, F.; ARAKAWA, A. Numerical Methods Used in Atmospheric Models. GARP Publications Series, $\mathrm{N}^{\circ} 17$, Volume I, 62p, 1976.

MENÉNDEZ, C.G.; SAULO, A.C.; LI, Z.-X.Simulation of South American wintertime climate with a nesting system. Climate Dynamics, v. 17, p. 219-231, 2001.

MISRA, V.; DIRMEYER, A.P.; KIRTMAN, B.P. Dynamic Downscaling of Seasonal Simulation over South American. Journal of Climate, v.16, p.103-117, 2003. 
MONCUNIL, D. F. et al. Uma breve descrição do sistema de previsão climática regionalizada (Downscaling Dinâmico) implantado na FUNCEME. In: Congresso Brasileiro de Meteorologia, 12.,Foz do Iguaçu, Brasil. Anais... 2002. v. 1, p. 1291-1296. CD- ROM. (INPE-9505PRE/5158). Disponível em: <http://urlib.net/sid.inpe.br/ iris@1915/2005/04.13.17.28>. Acesso em: 17 abr. 2013).

MOREIRA, D. S. et al. Coupling between the JULES land-surface scheme and the CCATT-BRAMS atmospheric chemistry model (JULES-CCATT-BRAMS1. 0): applications to numerical weather forecasting and the $\mathrm{CO} 2$ budget in South America. Geoscientific Model Development Discussions, v. 6, p. 453-494, 2013.

NICOLINI, M.; SALIO, P.; KATZFEY, J.J.; McGREGOR, J.L. January and July regional climate simulation over South American. Journal of Geophysical Research, v.107, p. 4637, 2002.

NOBRE, P.; A. D. MOURA.; L. SUN. Dynamical downscaling of seasonal climate prediction over northeast Brazil with ECHAM3 and NCEP'S Regional Spectral Model at IRI. Bulletin of the American Meteorological Society, v.82, p.2787-2796, 2001.

OYAMA, M. D. Erros sistematicos no clima da regiao tropical da America do Sul simulado pelo modelo regional MM5 em baixa resolucao horizontal. Revista Brasileira de Meteorologia, v. 21, n. 1, p. 118-133, 2006.

PIELKE, R. A. Mesoscale meteorological modeling. Academic Press. 1984. 612p.

PIELKE, R. A. et al. A comprehensive meteorological modeling system -RAMS. Meteorology and Atmospheric Physics, v. 49, p. 69-91, 1992.

QIAN, J.-H.; SETH, A.; STEPHEN, Z. Reinitialized versus Continuous Simulation for Regional Climate Downscaling. Monthly Weather Review, v.131, p.2857-2874, 2003.

REYNOLDS, R. W.; RAYNER, N. A.; SMITH, T. M.; STOCKES, D. C.; WANG, W. An improved in situ and satellite SST analysis for climate. Journal of Climate, v. 15, p. 1609-1625, 2002.

ROPELEWSKI, C. F.; HALPERT, M. S. North American precipitation and temperature patterns associated with the El Niño/Southern Oscillation (ENSO). Monthly Weather Review, v. 114, n. 12, p. 2352-2362, 1986.

ROPELEWSKI, C. F.; HALPERT, M. S. Precipitation patterns associated with high index phase of Southern Oscillation. Journal of Climate, v. 2, n. 3, p. 268-284, 1989.
ROSÁRIO, N. E.; LONGO, K. M.; FREITAS, S.; YAMASOE, M. A.; FONSECA, R. M. Modeling South America regional smoke plume: aerosol optical depth variability and shortwave surface forcing. Atmospheric Chemistry Physics Discuss, v. 12, p. 17465-17501, 2012.

SANTOS, A. F. et al. A parametric study for firefly algorithm by solving an inverse problem for precipitation field estimation. In: 1st International Symposium on Uncertainty Quantification and Stochastic Modeling (Uncertainties 2012), 2012, Maresias, SP. Proceedings of the $1 \mathrm{st}$ International Symposium on Uncertainty Quantification and Stochastic Modeling, 2012.

SEN, O.L.; WANG, Y.; WANG, B. Impact of Indochina deforestation on the East-Asian summer monsoon. Journal of Climate, v. 17, p. 1366-1380, 2004a.

SEN, O.L.; WANG, Y.; WANG, B. Re-greening the desertification lands in northern China: Implications from a regional climate model experiment. Journal of Meteorological Society of Japan, v.82, n. 6, p. 1679-1693, 2004b.

SETH, A.; ROJAS, M. Simulation and sensitivity in a nested modeling system for South American. Part I: Reanalyses boundary forcing. Jornal of Climate, v.16, p. 2437-2453, 2003.

SILVA, S. B. V., KOUSKY, E. V., SHI, W., HIGGINS, W. An Improved Grided Historical Daily Precipitation Analysis for Brazil. Journal Hydrometeorology, v. 8, p. 848-861, 2007.

SOUZA, E. P. Estudo teórico e numérico da relação entre convecção e superfícies heterogêneas na região amazônica. 1999. 121 p. Tese (Doutorado em Meteorologia) - Universidade de São Paulo, São Paulo, 1999.

SUN, L.; MONCUNILL, D. F.; LI, H.; MOURA, A. D.; SOUZA FILHO, F. A. Climate downscaling over northeast Brazil using NCEP RSM97. Journal of Climate, v.18, n. 4, p.551-567, 2005.

WALKO, R. L.; TREMBACK, J. C.; HERTENSTEIN, R. F. A. RAMS-The regional atmospheric modeling system, version 3b, user's guide. ASTER Division, Mission Research Corporation, Fort Collins, CO, 1995.

WILLIAMS, P. D. A proposed modification to the RobertAsselin time filter. Monthly Weather Review,v. 137, p. 2538-2546, 2009. 